\title{
Value of advance care planning and quality palliative care for patients nearing end-of-life
}

\section{Rhea Go-Coloma* \\ LMSW, Chief Administrative Officer, Hospice of the West, USA}

Poor prognoses, the loss of functional capabilities, and the need for advanced care planning are just some of the emotionally charged challenges of caring for individuals with advanced illness. These issues are especially relevant for case managers seeking to meet the complex needs of these patients as they near end-of-life.

Fortunately, a new approach to advance care planning (ACP) and palliative care transcends the idea of "giving in" to death and focuses instead on providing supportive care and decision-making as a vital part of delivering palliative care services that relieve socio-economic burdens for patients and families.

This is significant, given current health and population trends. By the year 2050, the proportion of the U.S. population that is over the age of 65 will increase to 20.3 percent, a factor that will impact the organization and delivery of healthcare. With the shift in focus from acute to chronic illnesses, and the need to transition from one-time interventions that correct a single problem to the ongoing management of multiple diseases and disabilities, a growing number of case managers are seeking innovative approaches to caring for patients with advanced illness.

\section{The role of case managers with palliative care}

While traditionally grouped with hospice care, palliative care takes a more holistic approach to supporting patients and caregivers facing serious illnesses. Palliative care offers psycho-social support that may have gone missing from other care models and plays a pivotal role in addressing the needs of the whole person and the family, improving quality of life for all. It can be provided along with curative treatment, offering services that effectively address the symptoms and stress of advanced illnesses.

The New England Journal of Medicine published research showing that palliative care can help patients live longer: in a study of 151 patients with advanced lung cancer, those given early palliative care survived 11.6 months, nearly three months longer than those who received standard medical care [1].

The goal is to treat the whole patient collaboratively and match treatment options with personal wishes. This often poses a big hurdle because people's end-of-life preferences are frequently unknown, and their manner of death is rarely in keeping with their individual desires. For case managers, this level of service may be an important component in the care management continuum.

For instance, 90 percent of adults say they would prefer to receive end-of-life care in their home if they were terminally ill, yet data show that only about one-third of Medicare beneficiaries (age 65 and older) died at home [2].

\section{Communicating goals of care, establishing advance directives}

Community-based palliative care helps individuals and families make care decisions based upon their own values, goals and preferences. This approach not only helps patients to manage symptoms and more easily deal with pain, depression and other issues, but it also enhances patient and family satisfaction with care. Again, these are key concerns for case managers as they strive to coordinate care that better addresses patient expectations and needs.

Palliative care specialists can ensure that patients get the care they need to avoid unnecessary hospitalization or unwanted care, another key objective for case management. This approach helps to lower the cost of care by reducing visits to the emergency department, ICU days and in-hospital stays, resulting in lower out-of-pocket expenses for both patients and family caregivers.

\section{The value of advance care planning and advance directives}

Consider this case for example: a female patient, 89 , who had a history of heart failure and spinal stenosis, lived at home with her elderly husband. In a period of months, she was admitted twice to the hospital with symptoms of heart failure. Her Medicare Advantage plan used a predictive algorithm to identify her as a candidate for homebased palliative care. When seen at home on the first visit, the palliative nurse discussed with her specific goals of care.

Her preference was to avoid aggressive care and hospitalization, and to disable her implantable cardioverter defibrillator -- decisions that were documented in an advance directive $(\mathrm{AD})$, legal documents that instruct physicians on how to carry out medical decisions. ADs are essential for ensuring that a patient's wishes for end-of-life care will be carried out. ADs allow people to communicate with doctors, relatives and friends when they are unable to do so during a medical crisis. This includes Durable Power of Attorney for Healthcare, a Living Will, and a form confirming the Physician Orders for Life-Sustaining Treatment.

It's important to keep in mind that end-of-life care plans and decisions must be tailored to each individual. Ideally, instructions expressing one's care wishes should be part of everyone's personal portfolio of documents. This is an important component of palliative

Correspondence to: Rhea Go-Coloma, LMSW, Chief Administrative Officer, Hospice of the West, USA, E-mail: ndufour@cpronline.com

Received: February 12, 2018; Accepted: February 19, 2018; Published: February 23,2018 
care: ensuring that the person's wishes are respected while relieving stress, diminishing anxieties, and avoiding disputes among loved ones. It is also a priority concern for case managers.

Despite the need and critical importance of ADs, only 25 percent of Americans have recorded their end-of-life medical wishes in a legal document. This pervasive lack of advance care planning presents a problem because more than half of patients are unable to participate in end-of-life decisions when the time comes [3].

Whether or not an $\mathrm{AD}$ is in place, the goal is for providers to understand patients' wishes on how they want to die, and whether treatment should be continued-and under which circumstances-or whether treatment should be ceased.

\section{Population health management and specialized palliative care}

Successful models of palliative care require clinically driven programs, as well as the proper infrastructure, staffing and resources to intervene when something is predicted to happen to patients facing advanced illness.

Case managers should be aware of the introduction of new population health management solutions that include an innovative model for this specialized level of palliative care. By providing highly structured and consistent clinical programming in combination with quality monitoring and oversight, it's a unique opportunity to offer program focused specifically for this advanced illness population. With emphasis on solutions that meet the explicit challenges of those members (and their caregivers) facing serious illnesses, these programs are meeting wide receptivity.

The model begins by utilizing sophisticated analytics to identify members who are high opportunity -- not simply high cost - in advance of the last six months of life. It is the timeframe before patients undergo non-beneficial treatments and technology that is frequently offered by default, and too often results in over-medicalized deaths marked by costly hospitalizations.

The model then takes the important step of convening communitybased palliative care teams comprised of nurses and clinical social workers practicing at the top of their licensure, and who typically reside as a separate service within local hospice and palliative care organizations. These teams document member engagement activities in a platform and follow structured risk-based care pathways and care management protocols.

They rely on comprehensive, standardized, baseline and followup palliative phone and home visit questionnaires to perform and document initial and follow-up patient assessments. The team reviews symptoms, performs medication reconciliation, and discusses and documents goals of care. They guide advance care planning, provide psychosocial support and identify caregiver needs.
These clinicians then create a palliative plan of care based upon patient goals, while providing ongoing support for enhancing home supports, providing patient education and assisting with patient decision-making.

\section{Taking the lead}

When considering an innovative approach such as the one outlined, case managers should appoint a clinical team leader, or liaison, as someone who can function effectively with a clear understanding of organizational and leadership structure. As the disease progresses, palliative care specialists might take a more central role in the patient's care in collaboration with the physician who leads the patient's medical home. Research has shown success in, for example, heart failure where a case management nurse communicated with patients and delegated responsibility for different aspects of care [4].

This approach advances coordination of care, establishes a team contact and a source of trust and comfort for patients and caregivers. The clinical team leader is then better able to assure that medical decision-making is tailored to patients' values, goals and preferences [5].

\section{Helping patients live longer}

The goal of palliative care is to improve quality of life for patients at any stage of illness regardless of current treatment plans and is tailored to the needs of the patient and the family.

Palliative specialists represent a natural go-to source of professional talent that makes it possible for home-based palliative care programs to match the appropriate resources to individual needs and scale the programs for large populations. When they are equipped with the technology, tools and training required to decrease variability in care, they help to drive positive outcomes.

These clinicians effectively interact and coordinate care with the designated physician, case manager and others designated as responsible for the patient's ongoing medical care. This collaborative approach advances care coordination, reduces the cost of care, and results in greater patient/caregiver satisfaction.

\section{References}

1. Temel JS, Greer JA, Muzikansky A, Gallagher ER, Admane S, et al. (2010) Early Palliative Care for Patients with Metastatic Non-Small-Cell Lung Cancer. $N$ Engl $J$ Med 363: 733-742. [Crossref]

2. Teno JM (2013) Approaching Death: Improving Care at the End of Life, Institute of Medicine, "Change in End-of-Life Care for Medicare Beneficiaries". JAMA 309: 470-477.

3. Demoratz, Michael. Advance directives in end of life care. Aging Care. Accessed August 31, 2017.

4. https://www.agingcare.com/articles/advance-directives-in-end-of-life-care-188604.htm

5. Aiken LS, Butner J, Lockhart CA, Volk-Craft BE, Hamilton G, et al. (2006) Outcome evaluation of a randomized trial of the PhoenixCare intervention: program of case management and coordinated care for the seriously chronically ill. $J$ Palliat Med 9: 111-126. [Crossref]

Copyright: (C2018 Go-Coloma R. This is an open-access article distributed under the terms of the Creative Commons Attribution License, which permits unrestricted use, distribution, and reproduction in any medium, provided the original author and source are credited. 